\title{
Wind-pollinated Plants of Proven Allergenicity and of Greatest Clinical Importance
}

\section{TREES}

Birch family
Alder
Birch
Hazel
Beech family
Beech
Oak
Elm family
Elm
Hackberry
Maple family
Box elder
Maple
Sycamore family
Plane tree (sycamore
Poplar-willow family
Aspen
Cottonwood
Poplar
Willow
Walnut-hickory family
Hickory
Pecan
Walnut
Olive family
Ash
Olive

Casuarina (beefwood) family Australian pine
Betulaceae

Alnus

Betula

Corylus

Fagaceae

Fagus

Quercus

Ulmaceae

Ulmus

Celtis

Aceraceae

Acer negundo

Acer

Platanaceae

Platanus

Salicaceae

Populus

Populus

Populus

Salix

Juglandaceae

Carya

Carya

Juglans

Oleaceae

Fraxinus

Olea

Casuarinaceae

Casuarina 


\section{GRASSES}

Grass family

Bermuda grass

Bluegrass

Orchard grass

Red top

Rye grass

Sweet vernal grass

Velvet grass
Gramineae

Cynodon dactylon

Poa pratensis

Dactylis glomerata

Agrostis alba

Lolium spp.

Anthoxanthum odoratum

Holcus lanatus

\section{WEEDS}

Sunflower family

Ragweeds

Marsh elder

Cocklebur

Sagebrush and wormwood

Pigweed (carelessweed) family

Goosefoot family

Saltbush

Plantain family

Plantain

Hemp family

Hemp

Knotweed family

Sorrel

Dock
Compositae

Ambrosia

Iva

Xanthium

Artemisia

Amaranthaceae

Chenopodiaceae

Atriplex

Plantaginaceae

Plantago

Cannabinaceae

Cannabis

Polygonaceae

Rumex

Rumex 